\title{
Treating pediatric metastatic neuroblastoma with chemotherapy-based multimodality approach in a non-transplant setting - experience from a developing country
}

\author{
Manjusha Nair ${ }^{1}$, Prasanth V R ${ }^{1}$, Priyakumari Thankamony ${ }^{2}$, Binitha Rajeswari ${ }^{1}$, \\ Guruprasad C S ${ }^{3}$, and Preethi George ${ }^{4}$ \\ ${ }^{1}$ Regional Cancer Centre Thiruvananthapuram \\ ${ }^{2}$ Regional Cancer Centre \\ ${ }^{3}$ Regional Cancer Center \\ ${ }^{4}$ Regional Cancer Centre Trivandrum
}

April 10, 2021

\begin{abstract}
Background: Children with metastatic neuroblastoma have inferior survival despite therapeutic advances. Myeloablative chemotherapy followed by stem cell transplantation, accepted as the current standard of care, is not accessible to patients in many developing countries due to resource constraints. We share our experience of treating metastatic neuroblastoma in a non-transplant facility with conventional chemotherapy, surgery, and radiotherapy. Method - Retrospective study of children 1-14years of age treated for metastatic neuroblastoma in our center from January 2008 to December 2017 Results - Eighty-nine patients with metastatic neuroblastoma received treatment. Mean age was 3.5years and male:female ratio was 1.1:1. The commonest primary site was suprarenal(55\%) and commonest site of metastasis was bone marrow(76\%). $40 \%$ patients had multiple metastatic sites. Mean baseline LDH was $3724 \mathrm{U} / \mathrm{L}($ range303-16609 U/L) and most(65\%) patients had LDH>750U/L.53 patients $(59.6 \%)$ had good response to chemotherapy as evidenced by clearance of metastatic disease, but out of them, 43 patients (81\%) progressed subsequently. 26 patients underwent surgery and 12 patients received maintenance therapy. 74 patients(86\%) developed recurrence and all but one died. Median time to recurrence and death were 9months(range 0-120months) and 10months(range 1-123months) respectively. At a median follow-up of 72months(range15-135months), 16 patients are alive, with 5 -year disease-free survival and overall survival of $17.6 \%$ and $18.4 \%$ respectively. Age, baseline LDH, chemotherapy regimen and response to treatment affected survival. Conclusion: Outcome of non-infant metastatic neuroblastoma remains dismal in a non-transplant setting. Younger age, lower baseline LDH and good response to chemotherapy appear to confer survival advantage, and may be used for risk-stratification in developing countries.
\end{abstract}

\section{TITLE PAGE}

\section{Manuscript Title:}

Treating pediatric metastatic neuroblastoma with chemotherapy-based multimodality approach in a nontransplant setting - experience from a developing country

Author List:

Manjusha Nair ${ }^{1}$,Prasanth V.R. ${ }^{1}$, Priyakumari T. ${ }^{1}$, Binitha Rajeswari ${ }^{1}$, Guruprasad C.S. ${ }^{1}$, Preethi Sara George ${ }^{2}$,

${ }^{1}$ Department of Pediatric Oncology, Regional Cancer Centre, Thiruvananthapuram, Kerala 
${ }^{2}$ Division of Cancer Epidemiology \& Biostatistics, Regional Cancer Centre, Thiruvananthapuram, Kerala Corresponding author:

Dr. Priyakumari Thankamony, MD, PhD

Professor and H.O.D,

Department of Pediatric Oncology,

Regional Cancer Centre,

Thiruvananthapuram,

Kerala-695011,

India

Ph: +919447032943

e-mail:drpriyarcc@gmail.com

Word Count for:

Abstract - 250

Main Text - 2204

Number of Tables -3

Number of Figures - 1

Running title - Metastatic Neuroblastoma in non-transplant setting

Keywords - pediatric, metastatic neuroblastoma, chemotherapy, survival, developing country.

Abbreviations key

\begin{tabular}{ll}
\hline NB & Neuroblastoma \\
\hline ASCT & Autologous stem cell transplantation \\
LDH & Lactate dehydrogenase \\
CT & Computed tomography \\
SPSS & Statistical Package for Social Sciences \\
DFS & Disease-free survival \\
OS & Overall survival \\
INRG & International Neuroblastoma Risk Group \\
SIOP PODC & International Society for Pediatric Oncology - Pediatric Oncology in Developing Countries \\
\hline
\end{tabular}

ABSTRACT

Background: Children with metastatic neuroblastoma have inferior survival despite therapeutic advances. Myeloablative chemotherapy followed by stem cell transplantation, accepted as the current standard of care, is not accessible to patients in many developing countries due to resource constraints. We share our experience of treating metastatic neuroblastoma in a non-transplant facility with conventional chemotherapy, surgery, and radiotherapy.

Method - Retrospective study of children 1-14years of age treated for metastatic neuroblastoma in our center from January 2008 to December 2017

Results - Eighty-nine patients with metastatic neuroblastoma received treatment. Mean age was 3.5years and male:female ratio was 1.1:1. The commonest primary site was suprarenal(55\%) and commonest site of 
metastasis was bone marrow(76\%). $40 \%$ patients had multiple metastatic sites. Mean baseline LDH was $3724 \mathrm{U} / \mathrm{L}($ range $303-16609 \mathrm{U} / \mathrm{L})$ and $\operatorname{most}(65 \%)$ patients had LDH $>750 \mathrm{U} / \mathrm{L} .53$ patients(59.6\%)had good response to chemotherapy as evidenced by clearance of metastatic disease, but out of them, 43 patients (81\%) progressed subsequently. 26 patients underwent surgery and 12 patients received maintenance therapy. 74 patients $(86 \%)$ developed recurrence and all but one died. Median time to recurrence and death were 9months(range 0-120months) and 10months(range 1-123months) respectively. At a median follow-up of 72months(range15-135months), 16 patients are alive, with 5-year disease-free survival and overall survival of $17.6 \%$ and $18.4 \%$ respectively. Age, baseline LDH, chemotherapy regimen and response to treatment affected survival.

Conclusion: Outcome of non-infant metastatic neuroblastoma remains dismal in a non-transplant setting. Younger age, lower baseline LDH and good response to chemotherapy appear to confer survival advantage, and may be used for risk-stratification in developing countries.

\section{INTRODUCTION -}

Neuroblastoma (NB), the most common pediatric extracranial solid tumour, is one of the most challenging childhood cancers to treat. Children with metastatic NB have inferior survival despite therapeutic advances like myeloablative chemotherapy and autologous stem cell transplantation (ASCT). These modern facilities are cost-prohibitive, and are not available in most of the public healthcare institutions in developing countries. In the non-transplant setting, metastatic NB is treated with conventional chemotherapy combined with local control modalities like surgery and/or radiotherapy. We determined treatment outcome and factors affecting survival of children over one year of age with metastatic NB treated at our centre with chemotherapy, surgery and radiotherapy.

\section{MATERIALS AND METHOD S}

Children 1-14years of age with Stage 4 NB treated at our centre over a 10-year period (1st January 2008 to 31st December 2017) were studied. Approval from Institutional review Board was obtained for the study. Clinical assessment for palpable disease, blood investigations including lactate dehydrogenase (LDH) and imaging of the primary site with ultrasound or computed tomography (CT) scan were done for disease evaluation. Metastatic workup included skeletal X-rays and bone marrow biopsy. The diagnosis of NB was established by histopathology and immunohistochemistry of bone marrow or primary tumour tissue.

Chemotherapy:

Based on the general condition, number of metastases, logistic and social factors and parental decision, patients received either of the two chemotherapy schemes derived from the St.Jude Neuroblastoma protocols. Chemo A was a moderately aggressive regimen consisting of vincristine $1.5 \mathrm{mg} / \mathrm{m} 2$, adriamycin $40 \mathrm{mg} / \mathrm{m} 2$ and cyclophosphamide $1500 \mathrm{mg} / \mathrm{m} 2$ alternating with cisplatin $100 \mathrm{mg} / \mathrm{m} 2$ and etoposide $450 \mathrm{mg} / \mathrm{m} 2$ every 3 weekly for one year (maximum cumulative dose of adriamycin $360 \mathrm{mg} / \mathrm{m} 2$ ). Chemo B was the less intensive regimen consisting of six 3 -weekly cycles of vincristine $1.5 \mathrm{mg} / \mathrm{m} 2$, adriamycin $30 \mathrm{mg} / \mathrm{m} 2$ and cyclophosphamide $750 \mathrm{mg} / \mathrm{m} 2$.

Response to chemotherapy:

Response assessment was done after 4 cycles of chemotherapy with bone marrow examination, skeletal xrays and imaging of the primary site. Disappearance of disease from metastatic sites was considered as good response.

Surgery and radiotherapy:

For patients who cleared the disease from metastatic sites, surgery was done if feasible, followed by further chemotherapy according to the assigned regimen. Radiotherapy was given for unresectable/residual disease after completion of intravenous chemotherapy regimen.

Maintenance Chemotherapy: 
In patients with stable disease after completion of chemotherapy, 6-8 months of oral metronomic chemotherapy was given with cyclophosphamide $50 \mathrm{mg} / \mathrm{m} 2$ and etoposide $50 \mathrm{mg} / \mathrm{m} 2$ daily for 20 days per month.

\section{STATISTICAL METHODS:}

The descriptive analysis included the absolute and relative frequency for categorical variables. Comparison between groups was carried out using the Chi-square test or Fisher's exact test. Variables for the survival analysis were age, primary site, metastatic site, baseline LDH, chemotherapy regimen, response to chemotherapy and surgery. The survival curve was estimated for each variable using the Kaplan-Meier method. The comparison between curves was obtained by the log-rank test. The Cox regression model was used to assess the effect of the variables on survival (multivariate analysis to calculate hazard ratios), which included variables with the following characteristics according to the Kaplan-Meier analysis with a significant difference $(\mathrm{p}<0.05)$. The level of significance established for all analyses was $5 \%$. All analyses were performed using the software SPSS@20.0 for Windows (Statistical Package for Social Sciences, IBM, USA).

\section{RESULTS}

Patient demographics:

There were 119 children $>1$ year of age with metastatic neuroblastoma, forming $50.2 \%$ of the total non-infant NB patients. Mean age of the patients was 3.5years (range 1-14 years) and male: female ratio was 1.1:1. 89 patients consented for treatment, and are included in the analysis.

Disease characteristics:

The most common site of primary tumour was suprarenal in 66 patients $(55.5 \%)$, followed by retroperitoneal $(\mathrm{n}=25,21 \%)$, thoracic/mediastinal $(\mathrm{n}=7,5.9 \%)$, cervical $(\mathrm{n}=8,6.7 \%)$ and multifocal $(\mathrm{n}=3,2.5 \%)$. Primary tumour was undetected in 6 patients (5\%). Commonest site of metastasis was bone marrow $(76.3 \%$ patients), followed by bone (15.9\%), lymph nodes (6.2\%) and liver (1\%). 36 patients (40.4\%) had multiple metastatic sites. Baseline LDH values were available for 79 patients, with mean value of $3724 \mathrm{U} / \mathrm{L}$ (range $303-16609$ U/L). 58 patients $(65 \%)$ had $\mathrm{LDH}>750 \mathrm{U} / \mathrm{L}$ and 21 patients $(23.5 \%)$ had $\mathrm{LDH}<750 \mathrm{U} / \mathrm{L}$.

Treatment and response:

Thirty-eight patients (42.6\%) received Chemo A, out of which 30 patients (78.9\%) had good response to chemotherapy and 6 patients $(15.7 \%)$ had poor response. 51 patients $(57.3 \%)$ received Chemo B, out of which 23 patients $(45.09 \%)$ had good response and 24 patients $(47.05 \%)$ had poor response. In 6 patients, response assessment could not be done because of early clinical progression or death. Surgery could be attempted in 26 patients (29.2\%), with excision in 16 patients, debulking in 6 patients and biopsy alone in 4 patients. Only 3 patients in this cohort received radiotherapy. 12 patients received metronomic maintenance chemotherapy after treatment completion (9 patients after Chemo A and 3 patients after Chemo B).

Table 1 shows the Chemotherapy regimen-based response and outcome

Relapse and death:

Overall 74 patients (86\%) developed recurrence/progression of disease. Out of the 53 patients who had good initial response to chemotherapy, 43 patients $(81 \%)$ relapsed. The median time to recurrence/progression was 9 months (range 1-120months). 73 patients (85.9\%) died, the median time to death being 10 months (range1-123months). Cause of death was disease progression in 71patients and toxicity-related deaths in 2 patients. 3 relapsed patients are lost for follow-up.

Survival:

At a median follow-up of 72 months (range 15-135months), there were 16 survivors. 5-year disease-free survival (DFS) was $17.6 \%$ and overall survival (OS) was $18.4 \%$. 
Mean age of survivors was 2.3 years (range 1-9 years) and their mean LDH at presentation was $728 \mathrm{U} / \mathrm{L}$ (range 303-1747 U/L). 11 patients out of 38 (28.9\%) who received Chemo A and 5 patients out of $51(9.8 \%$ ) who received Chemo B survived. Out of the 12 patients who received metronomic chemotherapy, 5 patients survived (41.6\%). (Table 2)

Prognostic factors:

In univariate analysis, $\mathrm{LDH}>750 \mathrm{U} / \mathrm{L}$, type of chemotherapy regimen, response to initial chemotherapy, number of chemotherapy cycles received and surgery were found to be statistically significant factors for DFS and OS. Age, primary site and number of metastatic sites were not statistically significant. On multivariate analysis, $\mathrm{LDH}>750 \mathrm{U} / \mathrm{L}$, Chemo B regimen and poor response to chemotherapy were statistically significant poor prognostic factors. (figure 1.)

Details of prognostic factors are given in Table 3.

\section{DISCUSSION:}

In the developing countries, a large number of children with NB present with metastatic disease, with frustratingly low survival even after skilful use of multiple treatment modalities. Many years back, in a study of 91 pediatric NB patients from our own centre, as high as 60\% children had Stage 4 disease, with long term survivors as low as $9 \%$ after chemotherapy, surgery and radiotherapy.[1]. Contemporary Indian studies by Bansal et al [2] and Agarwala et al [3] have also reported higher proportion of patients with advanced disease and poor survivals in NB treated with conventional non-myeloablative chemotherapy. Studies from other developing countries like Brazil and Turkey have reported 17\% and 6.5\% 5-year survival respectively for stage 4 NB under similar circumstances. [4], [5] In the high-income countries, accurate risk-stratification based on molecular diagnostics and advanced treatment facilities like high-dose chemotherapy followed by ASCT with excellent supportive care, surgery, radiotherapy and immunotherapy is able to yield around $40 \%$ survival in high-risk NB.[6] But limited availability and prohibitive cost of transplant facilities render them inaccessible to majority of the patients in resource-constrained settings like ours. The present study shows a trend towards improved survival over the years in children with metastatic NB treated without transplant, attributable to improvement in treatment facilities and supportive care.

Identifying clinical prognostic factors in this cohort of non-infant metastatic NB could guide us to develop a risk-adapted approach for treating these patients. Data from the International Neuroblastoma Risk Group (INRG) database reveal that clinical parameters like older age, involvement of bone marrow, bone and multiple metastatic sites are associated with worse outcome in metastatic NB. [7] The earlier studies from Children's Cancer Group had reported better outcome of Stage 4NB in children under 2 years of age and those with extraskeletal metastases alone. [8] In our study, children $<2$ years showed a trend towards better survival, but impact of age or the number of metastatic sites on survival was not statistically significant. Other well-known prognostic factors in metastatic NB supported by research are baseline LDH, ferritin and N-Myc amplification status.[6],[9] N-myc study was not done in our patients because of lack of facility within the institution. Ferritin could be estimated in only a few patients, and hence was not considered for analysis. LDH is a surrogate marker for N-myc amplification, $[6]$ and in the recently published INRG study, higher LDH at presentation had independent prognostic ability for worse DFS and OS in metastatic NB. [9] The SIOP PODC has recommended an arbitrary LDH value of $750 \mathrm{U} / \mathrm{L}$ as a prognostic marker when $\mathrm{N}$-myc status is not known. [6] In our cohort, patients with $\mathrm{LDH}>750 \mathrm{U} / \mathrm{L}$ demonstrated markedly inferior survival than those with $\mathrm{LDH}<750 \mathrm{U} / \mathrm{L}(18.2 \%$ vs $46.9 \%)$, and we plan to study prospectively its prognostic value in children with metastatic NB in our setting.

The question of whether intensity of chemotherapy improves survival in metastatic NB is a subject of various clinical trials. A retrospective analysis of 44 clinical trials by Cheung and Heller revealed that chemotherapy dose-intensity, particularly of cisplatin and teniposide, strongly correlated with clinical outcomes in metastatic NB.[10] In the present study, survival of patients who received the moderately intensive cisplatincontaining regimen A was far better than those who received the less aggressive regimen B (28.9\% vs 9.8\%), and the small group of patients who received 6-8 months of oral metronomic chemotherapy after the intensive 
regimen had prolonged survivals when compared to the whole cohort (41\% vs 18.4\%). However, a selection bias may have confounded the results, as patients with multiple metastases and poor general condition inadvertently received the less intensive chemotherapy regimen. Since cisplatin administration required staying in and around the hospital for frequent monitoring and subsequent supportive care, causing financial and social difficulties when cure was less likely, many parents opted out of this regimen during the earlier period of our study. Over time, with improvements in clinical facilities, human resources and availability of treatment assistance schemes, we were able to provide intensive chemotherapy and supportive care services for more patients, with minimal treatment-related mortality. This heterogeneity in selection of treatment regime may have influenced our results.

The relevance of good response to chemotherapy in metastatic NB in the non-transplant setting is uncertain because relapses occur early if not consolidated with ASCT.[3] In an earlier analysis from our own centre,15 out of 17children with metastatic NB treated with multiagent chemotherapy had a favourable response, but their 2-year survival was only $11.7 \%[11]$.In the present study, chemosensitivity was demonstrated in majority of the patients, but most of them relapsed and died within a short period (median 9 months and 10 months respectively) while continuing on the same chemotherapy regimen or soon after completing treatment. Whether further intensification of chemotherapy after getting a good initial response would have translated into more favourable outcomes in some of these patients is a matter worthy of consideration. A recently published study from India has reported survivals of around $30 \%$ in high-risk NB patients treated without ASCT using an intensive consolidation regimen with topotecan, vincristine and doxorubicin[12]

The impact of surgery on survivals of metastatic NB is debated. Several studies have demonstrated that radical resection of the primary tumour does not influence local control or outcome in non-infant metastatic $\mathrm{NB},[13]$ nevertheless, safe resection with or without local radiotherapy continues to be the accepted practice. In our study, patients who underwent tumour excision or debulking had significantly better outcome, but surgery and radiotherapy could be offered to very less number of patients.

Of interest are the characteristics of our survivor cohort. We found that they were younger(around 2 years of age), mostly female and presented with lower baseline LDH and mostly had limited metastases. Most of them received moderately intensive cisplatin-containing chemotherapy and all but one were good responders, and most underwent excision or debulking. This shows that interplay between multiple clinically demonstrable favourable prognostic factors may be contributory to better survival in children with metastatic NB treated without ASCT.

In conclusion, this study points out that outcome of metastatic NB patients remains suboptimal in a nontransplant setting, but there is a definite trend towards better survival over the years contributed by improvement in supportive care. Setting up enough transplant facilities to cater to the large numbers of metastatic NB patients in developing countries may not be practical because of the huge burden on resources. Under such circumstances, simple and affordable investigations like baseline LDH and response to initial chemotherapy may be useful in identifying selected patients who may have the chance of survival even without transplant. Younger patients with lower LDH at presentation may attain prolonged survival with moderately intensive cisplatin-containing chemotherapy, local treatment and metronomic maintenance chemotherapy. This knowledge may be utilised for justified allocation of the available resources so that patients with favourable prognostic factors are treated aggressively while those with adverse prognostic factors are spared the burden of treatment toxicity. Identification of patients expected to have poor survival may allow the focus to be directed on early provision of palliative care along with less intensive cancer-directed treatment aiming at reducing the symptom burden, improving the quality of life and smooth transition towards end-of-life care.

Acknowledgements: Nil

Conflict of interest: Nil

REFERENCES:

1. Kusumakumari P, Ajithkumar TV, Ratheesan K, Chellam VG, Nair MK. Pattern and outcome of 
neuroblastoma. A 10 year study. Indian Pediatr 1998; 35: 223-229.

2. Bansal D, Marwaha RK, Trehan A, Rao KL, Gupta V. Profile and outcome of neuroblastoma with conventional chemotherapy in children older than one year: a 15 y experience. Indian Pediatr. 2008;45:1359.

3. Agarwala S, Mandelia A, Bakhshi S, et al. Neuroblastoma: out- come over a 14 y period from a tertiary care referral centre in India. J Pediatr Surg. 2014;49:1280-5.

4. Lucena JN, Alves MTS, Abib SCV, Souza GO, Neves RPC, Caran EMM. Clinical and epidemiological characteristics and survival outcomes of children with neuroblastoma: 21 years of experience at the Instituto de Oncologica Pediatrica, in São Paulo, Brazil. Rev Paul Pediatr. 2018;36:254-260.

5. Aydn GB, Kutluk MT, Yalçn B, Büyükpamukçu M, Kale G, Varan A, Akyüz C, Senocak ME, Büyükpamukçu N. Neuroblastoma in Turkish children: experience of a single center. J Pediatr Hematol Oncol. 2009;31:471-80.

6. Parikh NS, Howard SC, Chantada G, Israels T, Khattab M, Alcasabas P, Lam CG, Faulkner L, Park JR, London WB, Matthay KK; International Society of Pediatric Oncology. SIOP-PODC adapted risk stratification and treatment guidelines: Recommendations for neuroblastoma in low- and middle-income settings. Pediatr Blood Cancer. 2015;62:1305-16.

7. Morgenstern DA, London WB, Stephens D, Volchenboum SL, Simon T, Nakagawara A, Shimada H, Schleiermacher G, Matthay KK, Cohn SL, Pearson AD, Irwin MS. Prognostic significance of pattern and burden of metastatic disease in patients with stage 4 neuroblastoma: A study from the International Neuroblastoma Risk Group database. Eur J Cancer. 2016; 65:1-10.

8. Schmidt ML, Lal A, Seeger RC, Maris JM, Shimada H, O'Leary M, Gerbing RB, Matthay KK. Favorable prognosis for patients 12 to 18 months of age with stage 4 nonamplified MYCN neuroblastoma: a Children's Cancer Group Study. J Clin Oncol. 2005;23:6474-80.

9. Moroz V, Machin D, Hero B, et al. The prognostic strength of serum LDH and serum ferritin in children with neuroblastoma: A report from the International Neuroblastoma Risk Group (INRG) project. Pediatr Blood Cancer. 2020;e28359. https://doi.org/10.1002/pbc.28359

10. Cheung NV, Heller G. Chemotherapy dose intensity correlates strongly with response, median survival, and median progression-free survival in metastatic neuroblastoma. J Clin Oncol. 1991;9:1050-8.

11. Kusumakumari P, Ajithkumar TV, Hariharan S, Varma RR, Chellam VG, Nair R, et al. Intensive chemotherapy in children with stage IV neuroblastoma. Indian J Pediatr 1999; 66: 867-872.

12. Richa Jain , Amita Trehan, Prema Menon et al. Survival in patients with high-risk neuroblastoma treated without autologous stem cell transplant or dinutuximab beta. Pediatr Hematol Oncol 2021;1-13. doi: 10.1080/08880018.2020.1850955.

13. Simon T, Häberle B, Hero B, Dietrich von Schweinitz, Berthold F. Role of surgery in the treatment of patients with stage 4 neuroblastoma age 18 months or older at diagnosis. J Clin Oncol. 2013 ;31(6):7528. doi: 10.1200/JCO.2012.45.9339. Epub 2013 Jan 2.

\section{Legends:}

TABLE 1 Response and outcome by treatment regimen

TABLE 2 Characteristics of the survivor cohort $(n=16)$.

TABLE 3 Prognostic variables on univariate analysis

FIGURE 1 Kaplan-Meier Survival Curves of a) OS by LDH $>750 \mathrm{U} / \mathrm{L}$ and $<750 \mathrm{U} / \mathrm{L}$ b) OS by type of chemotherapy regimen and c) OS by Response to chemotherapy

\section{Hosted file}

TABLE 1.pdf available at https://authorea.com/users/336955/articles/517472-treatingpediatric-metastatic-neuroblastoma-with-chemotherapy-based-multimodality-approach-ina-non-transplant-setting-experience-from-a-developing-country

\section{Hosted file}


TABLE 2.pdf available at https://authorea.com/users/336955/articles/517472-treatingpediatric-metastatic-neuroblastoma-with-chemotherapy-based-multimodality-approach-ina-non-transplant-setting-experience-from-a-developing-country

\section{Hosted file}

Table 3.pdf available at https://authorea.com/users/336955/articles/517472-treatingpediatric-metastatic-neuroblastoma-with-chemotherapy-based-multimodality-approach-ina-non-transplant-setting-experience-from-a-developing-country

\section{Hosted file}

Figure 1.pdf available at https://authorea.com/users/336955/articles/517472-treatingpediatric-metastatic-neuroblastoma-with-chemotherapy-based-multimodality-approach-ina-non-transplant-setting-experience-from-a-developing-country 